\title{
Restoration of out of focus images based on circle of confusion estimate
}

\author{
P. Vivirito ${ }^{* a}$, S. Battiato ${ }^{* a}$, S. Curti*a ${ }^{* a}$ M. La Cascia**b ${ }^{*}$, and R. Pirrone ${ }^{* * b}$ \\ ${ }^{\mathrm{a}} \mathrm{ST}$ Microelectronics, AST Catania Lab; ${ }^{\mathrm{b}} \mathrm{DIAI}$ - University of Palermo
}

\begin{abstract}
In this paper a new method for a fast out-of-focus blur estimation and restoration is proposed. It is suitable for CFA (Color Filter Array) images acquired by typical CCD/CMOS sensor. The method is based on the analysis of a single image and consists of two steps: 1) out-of-focus blur estimation via Bayer pattern analysis; 2) image restoration. Blur estimation is based on a block-wise edge detection technique. This edge detection is carried out on the green pixels of the CFA sensor image also called Bayer pattern. Once the blur level has been estimated the image is restored through the application of a new inverse filtering technique. This algorithm gives sharp images reducing ringing and crisping artifact, involving wider region of frequency. Experimental results show the effectiveness of the method, both in subjective and numerical way, by comparison with other techniques found in literature.
\end{abstract}

Keywords: Bayer pattern, circle of confusion, blur, blind restoration, inverse filtering, out-of-focus

\section{INTRODUCTION}

In the consumer electronics world the tendency is to provide an increasing number of functionality in a single unit. As a result, a mobile phone can acquire pictures and send them as e-mail messages through the cellular network. The images are acquired by CCD/CMOS sensor and processed by typical processing technique (Image Generation Pipeline) as described in [1]. However, due to limited dimension and quality of the camera inside the phone, images are often degraded. An enhancement of the acquired images is necessary in order to make them pleasant to the human viewer [2][3]. Our work is aimed to restore slightly out-of-focus images taken through low quality camera and lens. In this paper a new method for simultaneous out-of-focus blur estimation and restoration based on a single image is proposed. The method consists of two steps: 1) out-of-focus blur estimation; 2) image restoration by a new inverse filtering technique. Out-of-focus blur estimation and image restoration are two different image-processing problems. Blur estimation is used both for depth perception from two differently focused images of the same scene [4] and to estimate the out-of-focus blur of an image [5] [6].

The image restoration problem has been faced in several ways. NAS-RIF [7], for example, involves minimizing a cost function while NLIVQ (nonlinear interpolative vector quantization) [8] and ARMA methods [9] [10], are borrowed from data compression field. Others well known methods are blind deconvolution [11] and zero sheets algorithms [12]. A few of these methods require the knowledge of the Point Spread Function (PSF), others use statistical information. Others recent approaches like in [15], gives good results, but are not suitable for real time application.

Our work starts from consideration found in [13], [14], which propose complete blind restoration systems. In [13], analyzed images are subdivided in sub-blocks and an edge-detection algorithm, based on DCT coefficients, is applied. PSF is computed based on the average 1-D step response along the orthogonal direction of detected edges. Finally, a constrained least square filter is generated using the PSF. In [14] the authors use edge detection to estimate, via gradient analysis, the Line Spread Function (LSF). LSF is used to generate directly the PSF for constructing the power spectrum equalizer, based on Wiener filter [9].

The proposed method uses an extended DCT approach, (similar to the one described in [13]) for the edge detection, carried out only on half of the green pixels of the sensor image (Bayer pattern) [16]. A mean LSF is computed along gradient direction of detected edges. Using LSF is possible to compute the Circle-Of-Confusion (COC) diameter needed to obtain a geometrical optic PSF. Knowledge of PSF allows both simulating the acquisition process and constructing the proper filter. This way reduces storage and computational cost (obtaining also an higher acquisition rate).

* paolo.vivirito@st.com; phone: +39095 740 4278; fax: +39 095740 4004; STMicroelectronics, AST Catania Lab,

Stradale Primosole, 50 - 95121 Catania (CT), Italy; ** lacascia@unipa.it; phone: +39 091 481119; fax: +39 091 427940; Dipartimento di Ingegneria Automatica e Informatica, University of Palermo, Viale delle Scienze, 90128 Palermo (PA), Italy 


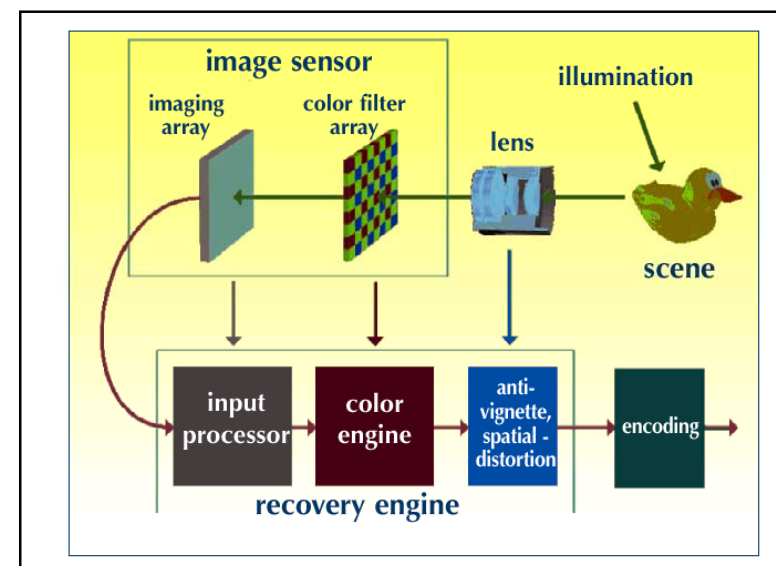

(a)

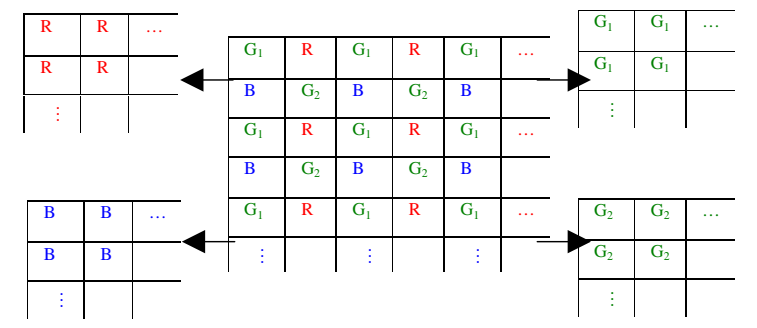

(b)

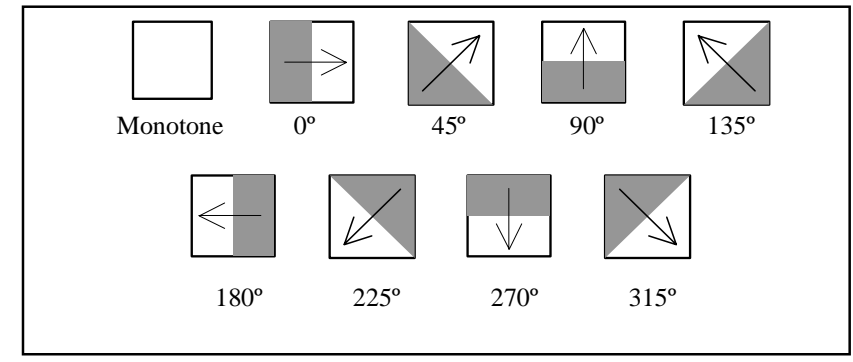

Figure 2. Proposed angle area subdivision.

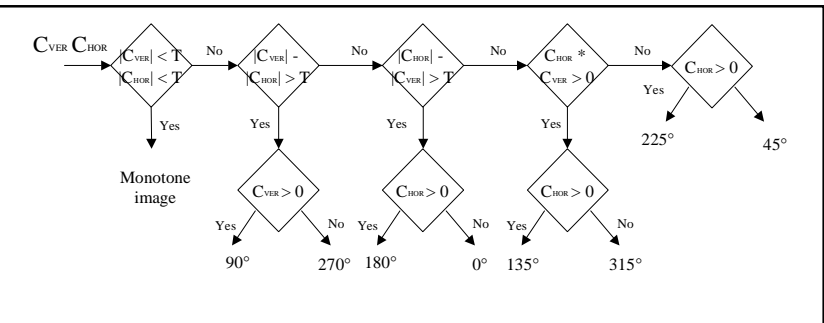

Figure 3. Edge classification.

Figure 1. (a) Image Acquisition Pipeline; (b) BP subsampling.

COC blur estimation is the most critical step, because it provides a measure of out-of-focus degree, with no knowledge of the particular camera system. In addition, such information is essential for correct generation of the restoration filter. The proposed restoration filter works onto wider region of frequency, compared with other inverse filtering algorithms, achieving sharp images and mitigating ringing and crisping effects. In order to evaluate the effectiveness of the method, results are compared to those obtained with the algorithms in [13] and [14].

The paper is structured as follows. Section 2 contains the description of COC estimation, PSF construction and the formulation of the proposed inverse filtering technique. Finally, experimental results and conclusions are presented in sections 3 and 4 .

\section{PROPOSED METHOD}

The image degradation process is assumed to be represented by a linear model [9]:

$$
g(s, t)=f(s, t) * h(s, t)+n(s, t)
$$

where $g(s, t)$ is the degraded image, $f(s, t)$ is the well-focused image, $h(s, t)$ is the PSF, $n(s, t)$ is the additive noise and * is the two-dimensional linear convolution operator.

The proposed method applies in the out-of-focus estimation phase directly on CFA images, but experiments proved that it is well- suited also for monochromatic images. Fig. 1 (a) shows the typical acquisition process. Bayer pattern is the output of the sensor acquisition process. CFA data are split in four homogeneous sub-matrixes: $G_{1}, G_{2}, R, B$ as shown in fig. 1 (b). In order to estimate the COC of the out-of-focus blur, LSF (line spread function) is used on $G_{1}$ (or $G_{2}$ ) channel obtained from BP splitting because it approximates the luminance component. Starting from estimated COC is possible to construct the $h(s, t)$ of (1), as much close as possible to the real one. At this point, the RGB image, obtained 
from the color engine, is first projected into a classical luminance/chrominance space, and then restored via inverse filtering.

\subsection{Out of focus estimation}

To evaluate the out-of-focus blur, the COC value is estimated starting from the $\mathrm{G}_{1}$ (or $\mathrm{G}_{2}$ ) channel of $B P$. The $\mathrm{G}_{1}\left(\right.$ or $\mathrm{G}_{2}$ ) channel is split in BxB blocks and for each $b_{k}$ block, the following DCT coefficients are computed [13]:

$$
\begin{aligned}
& C_{v e r}(k)=4 \sqrt{\frac{2}{B}} \sum_{n_{1}=0}^{B-1} \sum_{n_{2}=0}^{B-1} b_{K}\left(n_{1}, n_{2}\right) \cos \frac{\pi}{2 B}\left(2 n_{2}+1\right) \\
& C_{h o r}(k)=4 \sqrt{\frac{2}{B}} \sum_{n_{1}=0}^{B-1} \sum_{n_{2}=0}^{B-1} b_{K}\left(n_{1}, n_{2}\right) \cos \frac{\pi}{2 B}\left(2 n_{1}+1\right)
\end{aligned}
$$

$\mathrm{C}_{\mathrm{ver}}$ and $\mathrm{C}_{\mathrm{hor}}$ are respectively the correlation between the signal and a half period of cosine function in the vertical and horizontal axes. Gradient direction and versus are classified in eight areas, as shown in fig.2. If an edge is detected, its gradient is classified through the edge classification algorithm summarized in fig. 3, where $T$ is a suitable threshold, which determines when a significant contour is found. Let $s(r)$ be the generic one-dimensional step edge response along the direction of detected gradient. Let $a$ be the background intensity, $b$ the height of the step and $u(r)$ the standard unit step. For a degraded image:

$$
s(r)=h(r) *[a+b u(r)]
$$

where

$$
h(r)=\int_{0}^{2 \pi} h(r, \vartheta) d \vartheta
$$

is the LSF of the camera.

Since differentiation and convolution are linear operators the following equation holds:

$$
\frac{\partial s(r)}{\partial r}=h(r) * b \frac{\partial u(r)}{\partial r}=h(r) * b \delta(r)
$$

where $\delta(r)$ is the dirac delta function.

By definition of PSF:

$$
\int_{-\infty}^{+\infty} h(r) d r=1
$$

therefore:

$$
\int_{-\infty}^{+\infty} \frac{\partial s(r)}{\partial r} d r=b
$$

Thus, once a blurred edge is detected, the LSF can be obtained by means of [6]:

$$
h(r)=\frac{\frac{\partial s(r)}{\partial r}}{\int_{-\infty}^{+\infty} \frac{\partial s(r)}{\partial r} d r} .
$$


Let $l(r)$ be the averaged LSF of selected blocks, whose pattern is not monotone. It is reasonable to assume that $l(r)$ represents the LSF of the whole image. COC can be estimated by the following expression derived from geometrical optics:

$$
s=\frac{2 n}{\sqrt{1-\left(\frac{l(n)}{l(0)}\right)^{2}}}
$$

where $l(n)$ is the averaged LSF of selected blocks, $l(0)$ represents modal value of LSF, $n$ is the distance of examined $l(n)$ from modal value and $s$ is the COC diameter.

\subsection{Image restoration}

Starting from COC value, the PSF can be obtained, as described in [4], by:

$$
\operatorname{PSF}(r)= \begin{cases}\frac{4}{s^{2} \pi} \sqrt{s^{2}-4 r^{2}} & \mathrm{x} \leq \frac{\mathrm{s}}{2} \\ 0 & \text { otherwise. }\end{cases}
$$

Fourier transform of PSF, generates the "Optical Transfer Function" (OTF) of the optics system. In order to restore the out-of-focus image we propose this new power spectrum equalization (PSE) restoration filter:

$$
\operatorname{PSE}(u, v)=\frac{1}{\operatorname{OTF}(u, v) *\left[1+0.414\left(\frac{p}{|\operatorname{OTF}(u, v)|}\right)^{2}\right]}
$$

where $p$ represents the $3 \mathrm{Db}$ threshold of the restoration filter. This filter preserves phase components and allows mitigating noise enhancement and ringing artifacts related to classical filter peaks. As shown in figure 4(c), this approach respects the shape of optic MTF and limits, at the same time, the response of potentially noisy areas.

Let us consider now the three matrices $y(s, t), u(s, t)$ and $v(s, t)$ corresponding respectively, to luminance and chrominance components of the image, obtained from a process of color reconstruction (see [1] [17] [18] [19] for some details). The PSE restoration filter (12) is applied to the Fourier transform of $y(s, t), u(s, t)$ and $v(s, t)$ :

$$
\begin{aligned}
& Y^{\prime}(u, v)=Y(u, v) * \operatorname{PSE}(u, v) \\
& U^{\prime}(u, v)=U(u, v) * \operatorname{PSE}(u, v) \\
& V^{\prime}(u, v)=V(u, v) * \operatorname{PSE}(u, v)
\end{aligned}
$$

Restored color image is then obtained simply by back-transforming $Y^{\prime}(u, v), U^{\prime}(u, v)$ and $V^{\prime}(u, v)$ into the spatial domain. 


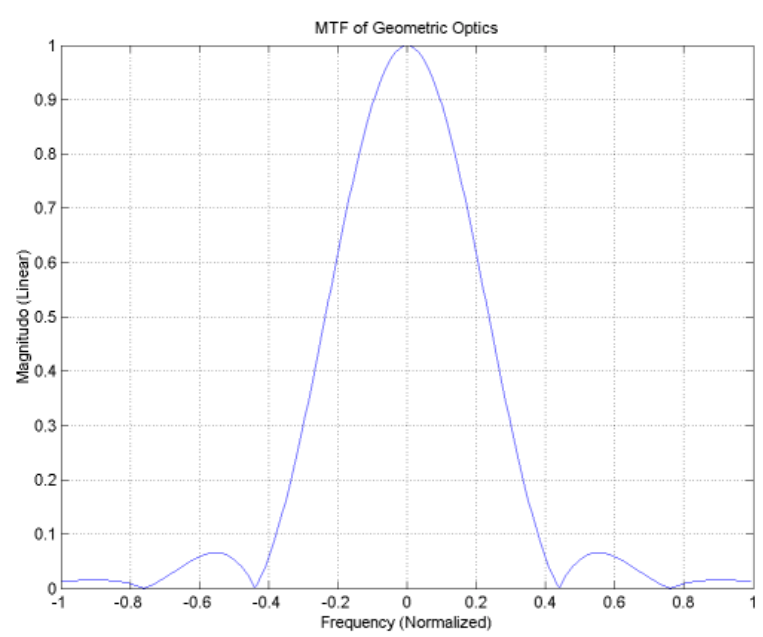

(a)

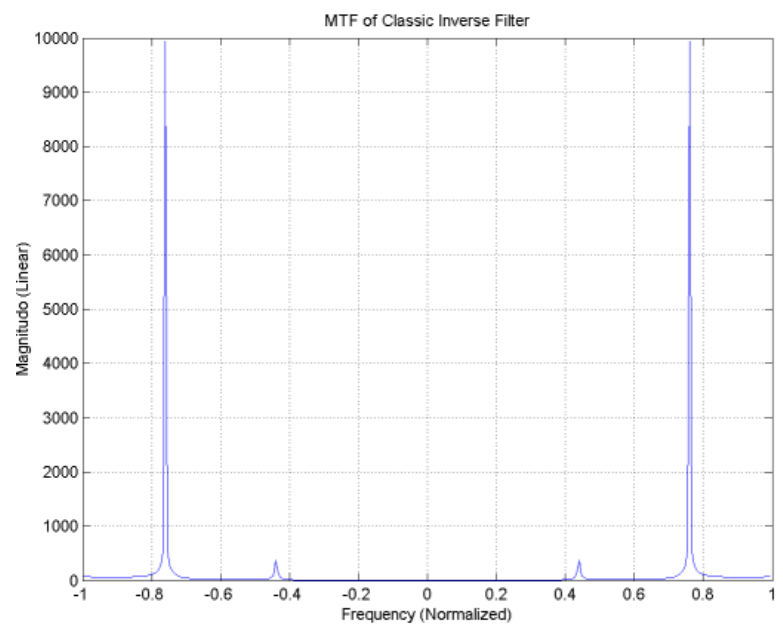

(b)

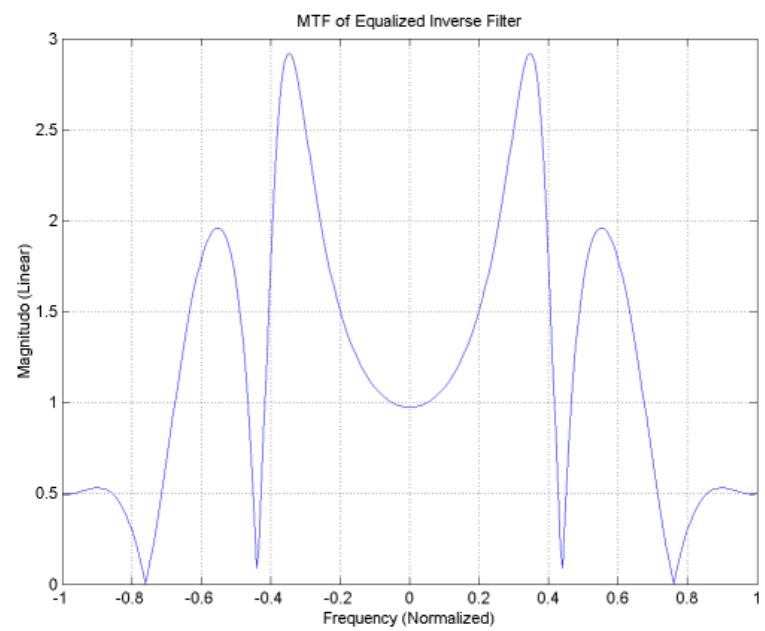

(c)

Figure 4. (a) MTF of a geometric optics; (b) MTF of a classic inverse filter approach; (c) resulting MTF of proposed approach.

\section{EXPERIMENTAL RESULTS}

In this section the effectiveness of the proposed method is evaluated by comparison with Kim [13] and Bhaskar [14] techniques. More specifically, results are compared subjectively, by image inspection both in spatial and frequency domain, and numerically, by evaluation of:

$$
q=\frac{l_{r}\left(r_{0}\right)}{l_{s}\left(r_{0}\right)} * \frac{d}{\sum\left[s_{s}(r)-s_{r}(r)\right]^{2}},
$$

where $s_{s}(r)$ and $s_{r}(r)$ are step response of well-focused and restored image at position $r, d$ is the number of evaluated pixels, $r_{0}$ is position of gradient peak, $l_{r}(r)$ and $l_{s}(r)$ represent, respectively, the gradient of sharp and restored image. The first half of (14) is the blurred-to-sharp gradient ratio [4], the right side is the inverse of mean square error of step 
response along direction of gradient (it is a measure of crisping and ringing effects). All images used in the experiments have been acquired by a 800 x 1000 CMOS sensor.

Figure 5 shows an example of application of the proposed method. It is clear that the restored image is much sharper than the blurred one, but it is a little noisier. Noise is related to the inverse filtering approach, but it is less emphasized in comparison to the state of art techniques [13] [14]. For sake of comparison with Bhaskar's and Kim's methods the experiments were conducted on gray level images. Fig. 6(a) shows a small area of an 800x1000 test image. Fig 6(b-d) present the results obtained respectively, with the proposed method, Bhaskar's method and Kim's method. Results reported in fig. 6(b) are obtained using $T=1000$ and $p=0.23$. Kim's method has been applied with $k=8$ and Cutoff $=450$ and Bhaskar's method with $k=30$ and threshold=0.05 (see [13] [14] for more details). Kim's method can be applied at all frequencies of the image, but it gives good results only for images with low defocus. Bhaskar's method is more efficient, but it introduces a strong aliasing in the images because it uses a fixed cutoff. The algorithm proposed in this paper leads to a good output, similar to Bhaskar, but without ringing artifact. Numerically, $q$ of (6) computed for images of fig. 6(b-d) is, respectively, 0.0434, 0.0056, and 0.0137 and that confirms the subjective evaluation.

Fig. 7(a-d) shows logarithmic spectra of images presented in fig. 6. Note that Kim's method works mainly on low and median frequencies, therefore sharp details are not improved. Bhaskar's method applies only onto small areas of frequency. The strong presence of aliasing in space domain can be explained by the sharp boundaries of regions where the filter is applied. The proposed algorithm works onto spectrum larger regions and their boundaries are smooth (see fig. 7(b)).

Preliminary research shows encouraging results using the filter directly in the CFA domain before the color interpolation. In figure 8 , a detail of such approach is presented.

\section{CONCLUSIONS}

A new blind deconvolution technique has been presented. It consists of two steps: COC blur estimation and image restoration. COC diameter is estimated by analyzing the edge gradient profile. Once COC is known, a geometric PSF is constructed to restore blurred image using a new inverse filtering formulation. Effectiveness of this method has been demonstrated by comparison with others inverse filtering techniques. Future works will include the possibility to evaluate the peculiarities of the proposed method in a well-defined optic-system. Also the capacity of working directly in the CFA domain will be further exploited.

\section{REFERENCES}

[1] M. Mancuso, S. Battiato, “An introduction to the digital still camera technology”, ST Journal of System Research, Vol. 2, No. 2, pp. 1-9, December 2001.

[2] S. Battiato, A. Bosco, M. Mancuso, G. Spampinato, " Temporal Noise Reduction of Bayer Matrixed Data "; Proceedings of IEEE ICME'02 International Conference on Multimedia and Expo 2002 - Lausanne, Switzerland, August 2002.

[3] S. Battiato, A. Castorina, M. Mancuso, "High Dynamic Range Imaging: Overview and Application", Accepted for publication SPIE Journal of Electronic Imaging, 2002.

[4] G. Schneider, B. Heit, J. Honig, J. Bremont, "Monocular depth perception by evaluation of the blur in defocused images", IEEE Proceedings of International Conference on Image Processing, Vol.2, pp. 116 -119, 1994.

[5] M. Subbarao, T.-C. Wei, G. Surya, "Focused image recovery from two defocused images recorded with different camera settings", IEEE Transactions on Image Processing, Vol.4, No.12, pp. 1613 -1628, Dec. 1995.

[6] A. Kubota, K. Kodama, K. Aizawa, "Registration and blur estimation method for multiple differently focused images", IEEE Proceedings of Proceedings of International Conference on Image Processing, Vol.2, pp. 515 -519, 1999.

[7] T.W.S. Chow, Xiao-Dong Li; S.-Y. Cho, "Improved blind image restoration scheme using recurrent filtering", IEE Proceedings on Vision, Image and Signal Processing, pp. 23-28, Feb. 2000.

[8] D.G. Sheppard, K. Panchapakesan, A. Bilgin, B.R. Hunt, M.W. Marcellin, " Removal of image defocus and motion blur effects with a nonlinear interpolative vector quantizer", IEEE Southwest Symposium on Image Analysis and Interpretation, pp. 1 -5, 1998.

[9] A. J. Jain, "Fundamentals of digital image processing", Prentice Hall International, Inc. 
[10] S. Chardon, B. Vozel, K. Chehdi, "A comparative study between parametric blur estimation methods", Proceedings of IEEE International Conference on Acoustics, Speech, and Signal Processing, Vol. 6, pp. 3233 3236, 1999.

[11] Lim J.S, "Two-dimensional signal and image processing”, Prentice Hall International, Inc.

[12] P. Premaratne, C.C. Ko, " Retrieval of symmetrical image blur using zero sheets", IEE Proceedings on Vision, Image and Signal Processing, Vol. 148 Issue 1, pp. 65 -69, Feb. 2001.

[13] S.K. Kim, S.R. Park, J.K. Paik, "Simultaneous out-of-focus blur estimation and restoration for digital autofocusing system", Electronic Letters, Vol.34, No.12, pp. 1217-1219, June 1998.

[14] R. Bhaskar, J. Hite, D.E. Pitts, "An iterative frequency-domain technique to reduce image degradation caused by lens defocus and linear motion blur", Geoscience and Remote Sensing Symposium, 1994. Surface and Atmospheric Remote Sensing: Technologies, Data Analysis and Interpretation, International, Vol.4, pp. 2522 2524, 1994.

[15] A. Jalobeanu, R.D. Nowak, J. Zerubia, M.A.T. Figueiredo, "Satellite and aerial image deconvolution using an EM method with complex wavelets", ICIP, Rochester, NY, USA, Oct. 2002

[16] B.E. Bayer, "Color Imaging array", U.S. Patent 3,971,065-1976.

[17] R. Kimmel, "Demosaicing image Reconstruction from color CCD samples", IEEE Transaction on Image Processing, Vol. 7, No. 3, 1999.

[18] R. Ramanath, "Interpolation methods for the Bayer color Array”, MS Thesis, North Carolina State University, 2000.

[19] H. J. Trussel, "Mathematics for Demosaicing”, To appear in IEEE Transanction on Image Processing, 2001.

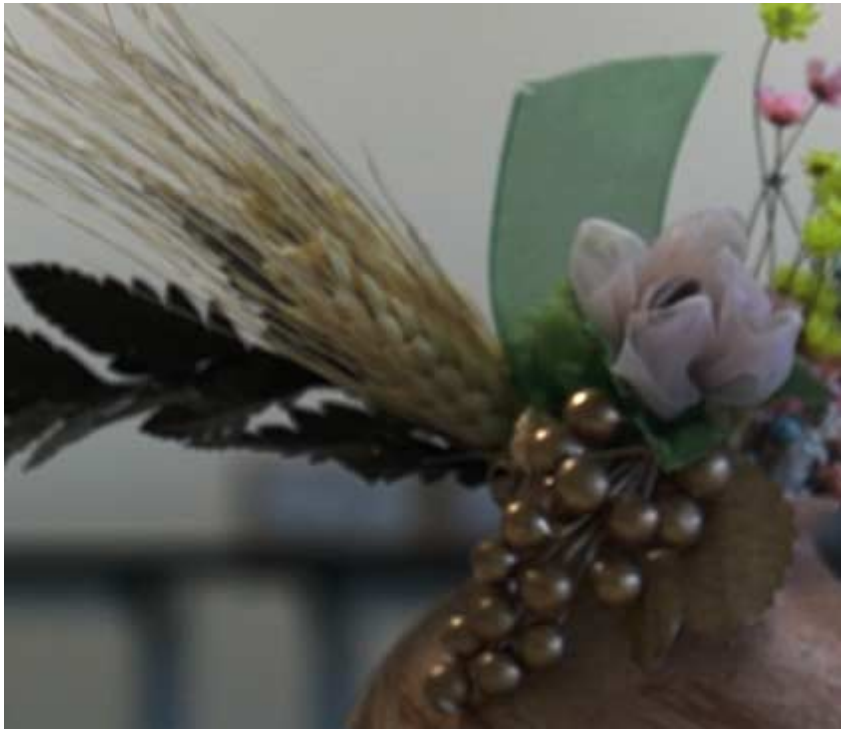

(a)

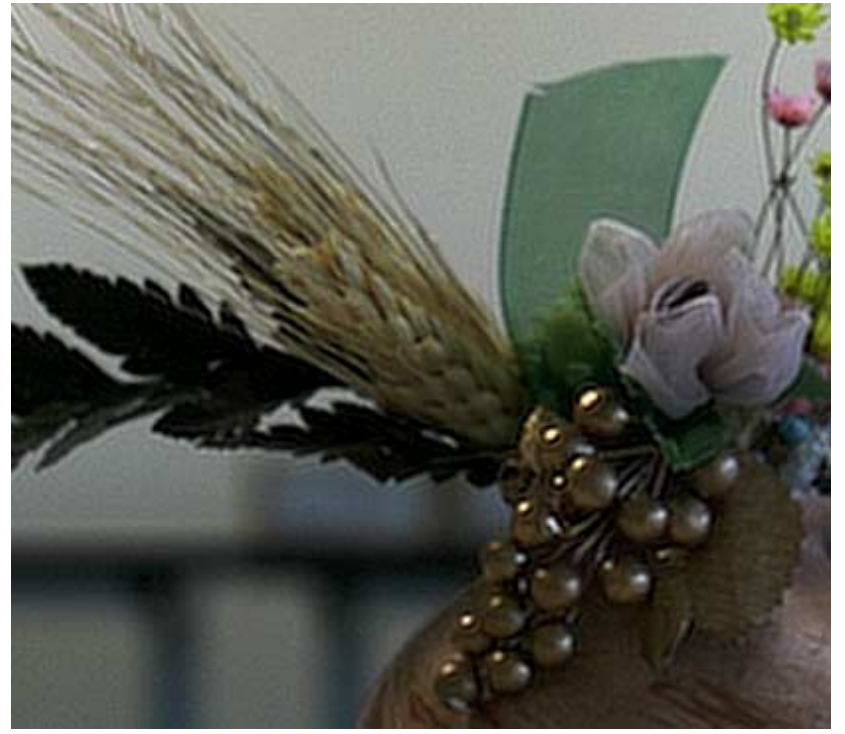

(b)

Figure 5. (a) Out-of-focus image; (b) Restored image. 


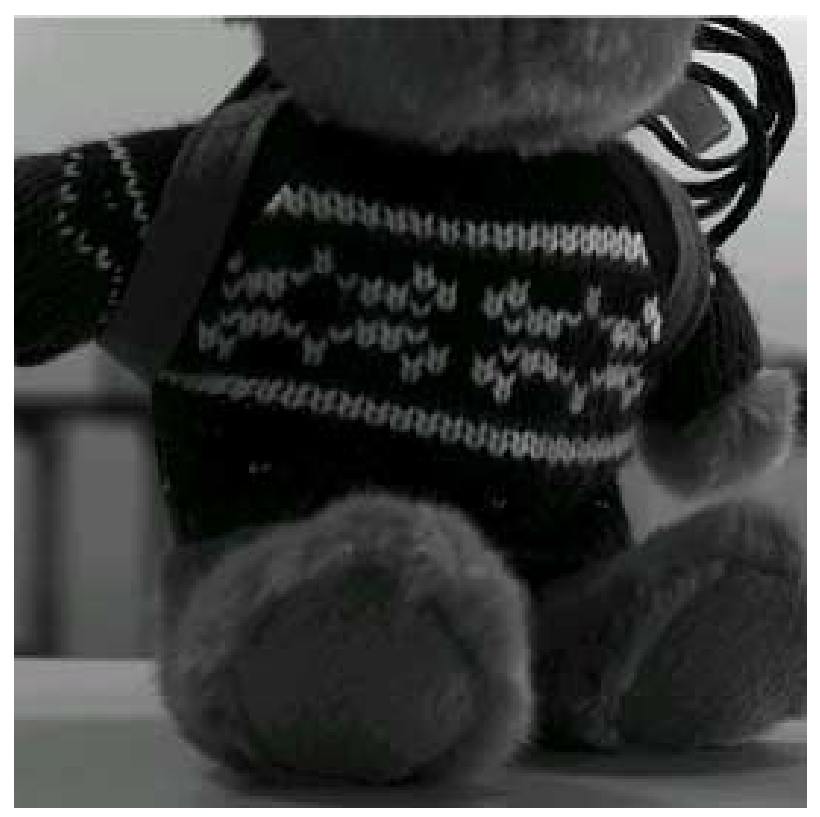

(a)

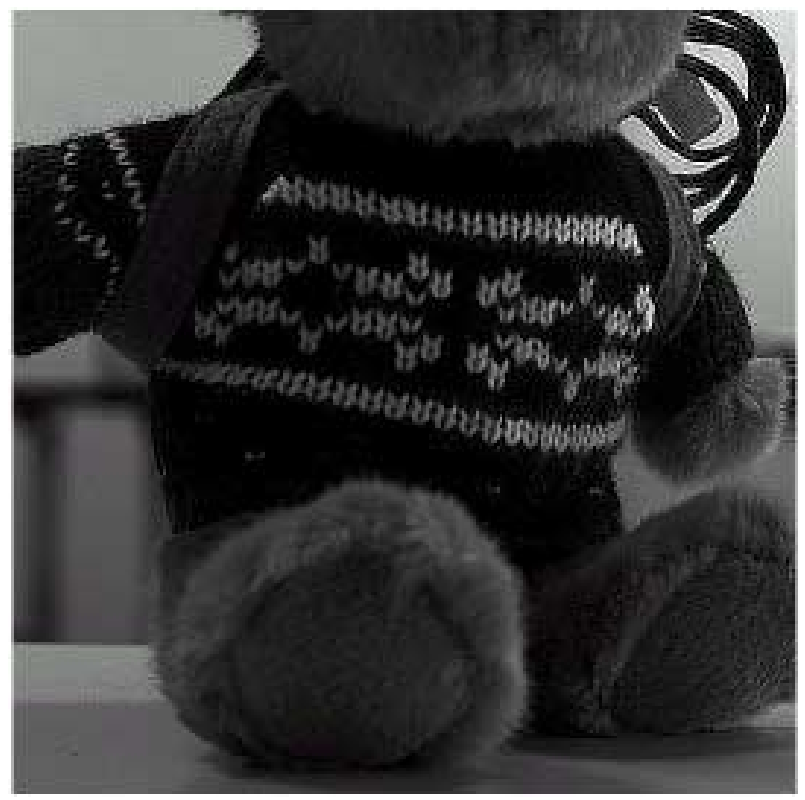

(c)

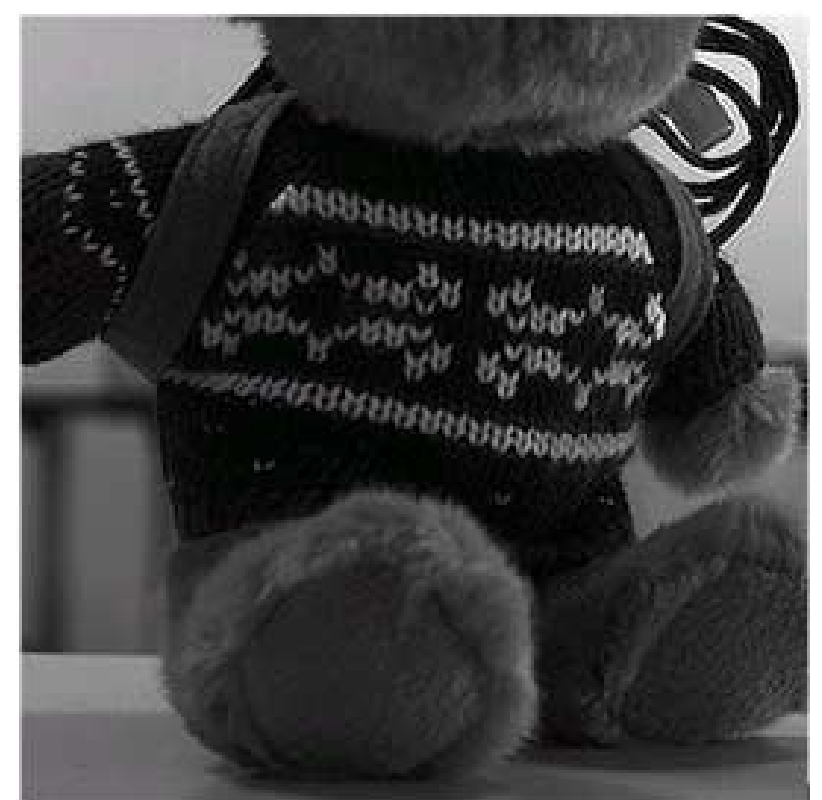

(b)

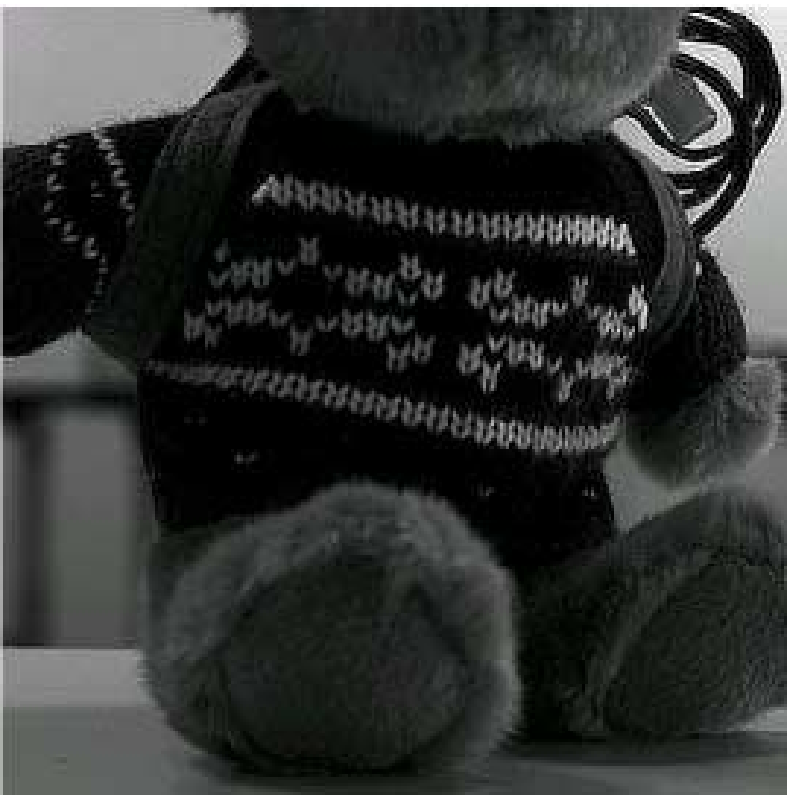

(d)

Figure 6. (a) Out-of-focus image; (b) Restored image using the proposed algorithm; (c) Restored image using Bhaskar's algorithm; (d) Restored image using Kim's algorithm. 


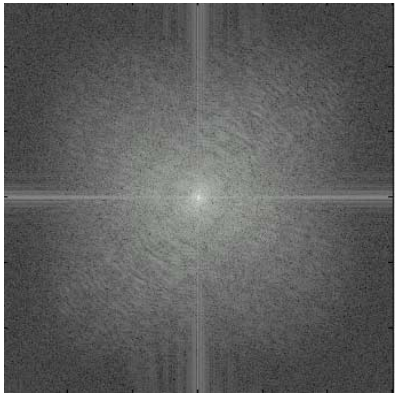

(a)

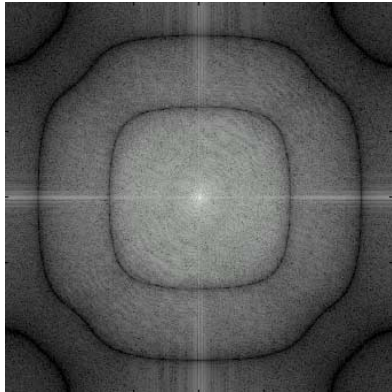

(b)

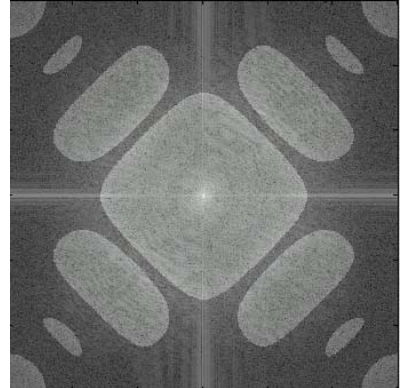

(c)

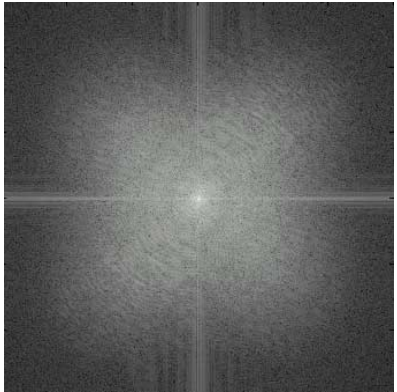

(d)

Figure 7. (a) - (d) Spectral representation of images in fig. 6.

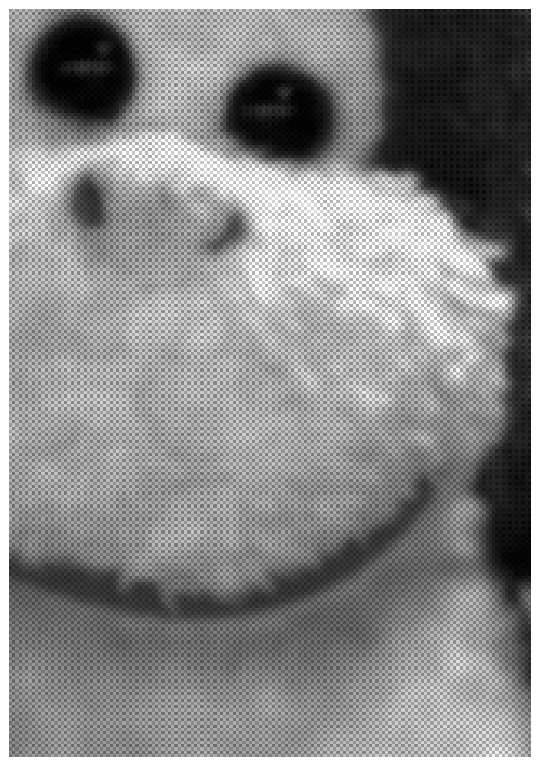

(a)

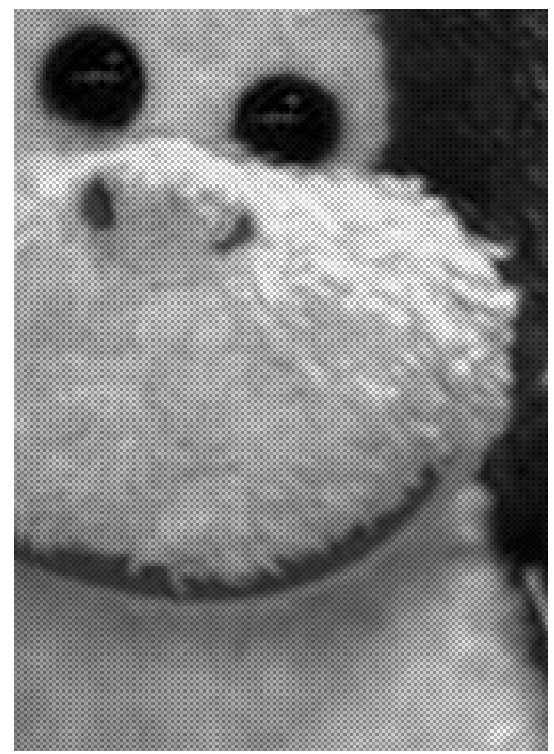

(b)

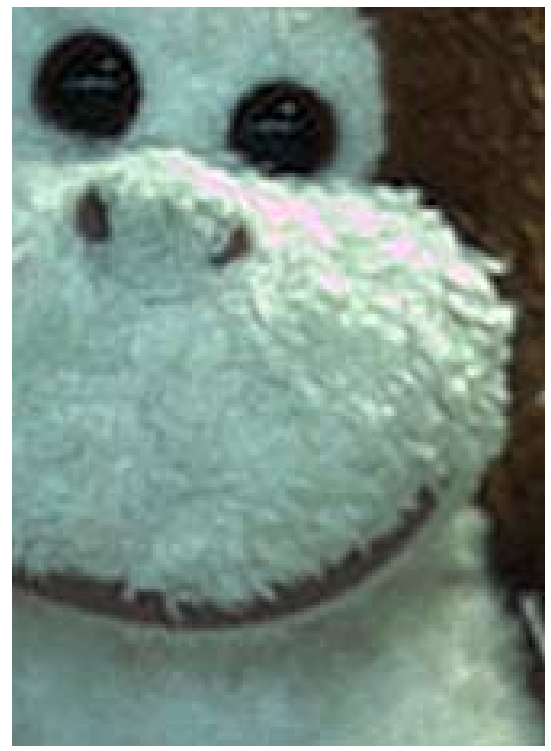

(c)

Figure 8. (a) Particular of an original CFA image, acquired by a CMOS sensor, slightly out-of-focus; (b) Restored image obtained applying the inverse filtering described in the paper, directly in the CFA domain; (c) The final color image obtained after a process of color reconstruction. The inverse filtering approach doesn't introduce noticeable artifacts color defects. 